\begin{tabular}{|c|c|c|}
\hline & $\begin{array}{c}\text { INTERNATIONAL JOURNAL } \\
\text { OF } \\
\text { PHARMACEUTICAL SCIENCES } \\
\text { RESEARCH }\end{array}$ & 䖧 \\
\hline
\end{tabular}

Received on 24 April, 2011; received in revised form 10 September, 2011; accepted 29 September, 2011

\title{
SYNTHESIS AND ANTICANCER ACTIVITY OF SOME NOVEL PYRIMIDINE DERIVATIVES
}

\author{
S. Sridhar* ${ }^{1}$, Y. Rajendra Prasad ${ }^{2}$ and S. C. Dinda ${ }^{3}$
}

Malla Reddy College of Pharmacy ${ }^{1}$, Secunderabad, Andhra Pradesh, India

University College of Pharmaceutical Sciences, Andhra University ${ }^{2}$, Vishakapatanam, Andhra Pradesh, India School of Pharmaceutical Education and Research, Berhampur University ${ }^{3}$, Berhampur, Orissa, India

Keywords:

Pyrimidines,

Synthesis,

Anticancer activity

Correspondence to Author:

Dr. S. Sridhar

H. No: 877 , Shyamala Nilayam, Near Vasantha Nagar colony Bus Stop, Vasantha Nagar, KPHB, Hyderabad,

Andhra Pradesh, India
ABSTRACT

A number of 2-amino-4-(2', 5'-dimethyl-3'-furyl)-6-(aryl)-pyrimidines 4(a-n) have been synthesized by treating the 1-(2', 5'-dimethyl-3'-furyl)-3-(aryl)-2propen-1-one (3a-n) with guanidine hydrochloride in presence of potassium hydroxide and ethanol. All these compounds were characterized by means of their IR, ${ }^{1} \mathrm{H}$ NMR spectroscopic data and microanalyses. When these compounds were evaluated for anticancer activity, some of them were found to possess significant activity.
INTRODUCTION: Pyrimidines comprise a relatively large, growing and most interesting group of antibacterial drugs which have made a major impact on the field of antibacterial chemotherapy particularly in the past few years. Many classes of chemotherapeutic agents containing pyrimidine nucleus are in clinical use such as antibacterial (sulfadiazines, sulfamerazine \& sulfamethazine), anticancer (5-fluorouracil and ftorafur), antiviral (iodoxuridine, trifluoridine and zidovudine), antifungal (flucytocine) and antimalarial agents (pyrimethamine) 1 .

Pyrimidines and their derivatives have been found to possess a broad spectrum of biological activities such as antimicrobial, anti-inflammatory, analgesic, antiviral and anticancer activities ${ }^{2-9}$. The synthesis of furan derivatives has engrossed substantial attention from organic and medicinal chemists for many years as they belong to a class of compounds with proven utility in medicinal chemistry ${ }^{10}$. Furan derivatives are known to be associated with multiple biological activities ${ }^{11,12}$. Therefore, both the pyrimidine and furan possess worthy and imperative bioactivities, which render them useful substances in drug research.

In view of these observations and in continuation of our research program on the synthesis of chalcones and their derivatives ${ }^{13,14}$, like pyrimidines, pyrazolines and isoxazolines, we report here in the synthesis of some new pyrimidine derivatives which have been found to possess an interesting profile of anticancer activity.

MATERIALS AND METHOD: Synthetic methods for the preparation of pyrimidine derivatives (4a-n) are summarized in scheme 1 . Chalcones were synthesized by the reaction of 3-acetyl-2, 5-dimethylfuran and various substituted aromatic and hetero cyclic aldehydes in presence of aq. $\mathrm{KOH}$ and ethanol. Pyrimidines were obtained in good yield by reacting chalcones (3a-n) with guanidine in presence of $\mathrm{KOH}$ and ethanol 15, 16. All the chemicals used in the synthesis were obtained from standard commercial sources. Melting points were determined in open capillaries, using Boitus melting point apparatus, expressed in ${ }^{\circ} \mathrm{C}$ and are uncorrected. 
Reactions were monitored by TLC using silica gel-G (Merck grade) as the adsorbent and the solvent systems are indicated at appropriate places. Silica gel (100-200 mesh, Merck grade) has been used for column chromatography. The ${ }^{1} \mathrm{H} N M R$ spectra of the compounds were recorded on Bruker AMX $400 \mathrm{MHz}$ NMR spectrophotometer using TMS as an internal standard and the values are expressed in $\delta \mathrm{ppm}$. Elemental analyses were carried out with a PerkinElmer model 2400 series II apparatus. The results of elemental analyses $(\mathrm{C}, \mathrm{H}$, and $\mathrm{N})$ were within $\pm 0.4 \%$ of the calculated values. All these compounds were also screened for their anticancer activity.

General procedure for preparation of 1-(2', 5'dimethyl-3'-furyl) -3-(aryl)-2-propen-1- one 3(a-n): A mixture of 3-acetyl-2, 5-dimethylfuran (0.005 mol) (1) and appropriate aldehyde $(0.005 \mathrm{~mol})$ (2a-n) was stirred in ethanol $(7.5 \mathrm{~mL})$ and then an aqueous solution of potassium hydroxide $(50 \%, 7.5 \mathrm{~mL})$ was added to it. The mixture was kept for $24 \mathrm{~h}$ and it was acidified with $1: 1 \mathrm{HCl}$ and $\mathrm{H}_{2} \mathrm{O}$. Then it was filtered under vacuum and the solid was washed with water, purified by column chromatography and crystallized from a mixture of ethyl acetate and hexane.

Synthesis of 2-amino-4-(2', 5'-dimethyl-3'-furyl)-6(aryl) pyrimidine (4a-n): 1-(2', 5'-dimethyl-3'-furyl)-3(aryl)-2-propen-1-one (3a-n) (0.001 mol) was condensed with guanidine hydrochloride $(0.001 \mathrm{~mol})$ in the presence of potassium hydroxide $(0.002 \mathrm{~mol})$ in absolute ethanol $(5 \mathrm{~mL})$ at reflux temperature on a water bath for $3 \mathrm{~h}$. The solvent was evaporated in vacuum and crushed ice was added to the residue while mixing thoroughly, whereupon a bright yellow solid separated out. This solid was filtered under vacuum, dried and purified by column chromatography to give pure pale yellow solid.
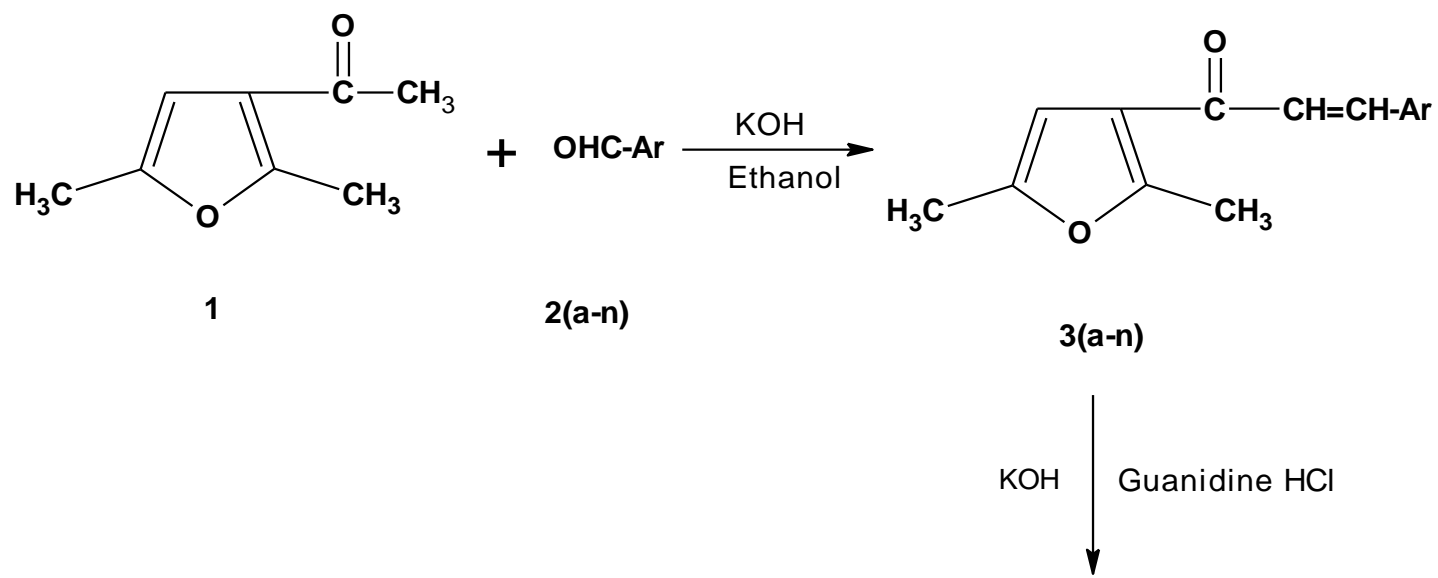

SCHEME 1

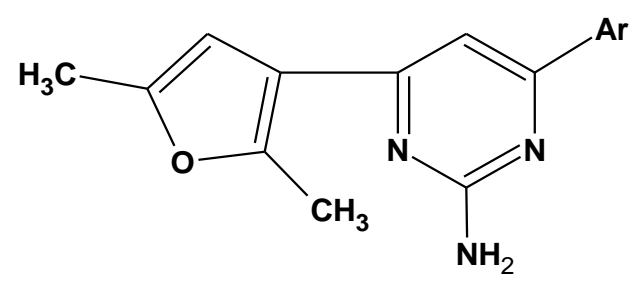

4(a-n)

TABLE 1: PHYSICAL DATA OF THE PREPARED COMPOUNDS 4 (a-n)

\begin{tabular}{|c|c|c|c|c|c|c|c|c|c|c|}
\hline \multirow{2}{*}{ S. No. } & \multirow{2}{*}{ Ar } & \multirow{2}{*}{ Mol. Formula } & \multirow{2}{*}{ M.P. $\left({ }^{\circ} \mathrm{C}\right)$} & \multirow{2}{*}{ Yield \% } & \multicolumn{3}{|c|}{ (\% Calc.) } & \multicolumn{3}{|c|}{ ( \% found) } \\
\hline & & & & & C & H & $\mathbf{N}$ & $\mathbf{C}$ & H & $\mathbf{N}$ \\
\hline $4 a$ & 3", 4", 5"-trimethoxyphenyl & $\mathrm{C}_{19} \mathrm{H}_{21} \mathrm{~N}_{3} \mathrm{O}_{4}$ & $312-314$ & 63 & 64.22 & 5.91 & 11.83 & 64.24 & 5.92 & 11.84 \\
\hline $4 b$ & 4"-chlorophenyl & $\mathrm{C}_{16} \mathrm{H}_{14} \mathrm{ClN}_{3} \mathrm{O}$ & $241-245$ & 57 & 64.21 & 4.68 & 14.01 & 64.24 & 4.69 & 14.11 \\
\hline $4 c$ & 4"-dimethylaminophenyl & $\mathrm{C}_{18} \mathrm{H}_{20} \mathrm{~N}_{4} \mathrm{O}$ & $164-165$ & 64 & 70.12 & 6.49 & 18.18 & 70.14 & 6.50 & 18.16 \\
\hline $4 d$ & 4"-methylphenyl & $\mathrm{C}_{17} \mathrm{H}_{17} \mathrm{~N}_{3} \mathrm{O}$ & $120-122$ & 54 & 73.11 & 5.01 & 15.05 & 73.14 & 5.03 & 15.15 \\
\hline $4 e$ & 2", 4"-dichlorophenyl & $\mathrm{C}_{16} \mathrm{H}_{13} \mathrm{Cl}_{2} \mathrm{~N}_{3} \mathrm{O}$ & $132-134$ & 60 & 57.65 & 3.90 & 12.61 & 57.62 & 3.87 & 12.64 \\
\hline
\end{tabular}




\begin{tabular}{ccccccccccc}
\hline $4 f$ & 9 "-anthracenyl & $\mathrm{C}_{24} \mathrm{H}_{19} \mathrm{~N}_{3} \mathrm{O}$ & $223-225$ & 72 & 78.90 & 5.20 & 11.50 & 78.92 & 5.21 & 11.54 \\
$4 \mathrm{~g}$ & 4"-methoxyphenyl & $\mathrm{C}_{17} \mathrm{H}_{17} \mathrm{~N}_{3} \mathrm{O}_{2}$ & $302-305$ & 68 & 69.15 & 5.76 & 14.23 & 69.14 & 5.75 & 14.22 \\
$4 \mathrm{~h}$ & 3", 4"-dimethoxyphenyl & $\mathrm{C}_{18} \mathrm{H}_{19} \mathrm{~N}_{3} \mathrm{O}_{3}$ & $220-221$ & 70 & 66.46 & 5.84 & 12.92 & 66.47 & 5.83 & 12.93 \\
$4 \mathrm{i}$ & 4"-fluorophenyl & $\mathrm{C}_{16} \mathrm{H}_{14} \mathrm{FN}_{3} \mathrm{O}$ & $233-235$ & 53 & 67.84 & 4.94 & 14.84 & 67.83 & 4.92 & 14.82 \\
$4 \mathrm{j}$ & 4"-nitrophenyl & $\mathrm{C}_{16} \mathrm{H}_{14} \mathrm{~N}_{4} \mathrm{O}_{3}$ & $260-262$ & 65 & 61.93 & 4.51 & 18.06 & 61.92 & 4.54 & 18.04 \\
$4 \mathrm{k}$ & 2"-pyridinyl & $\mathrm{C}_{15} \mathrm{H}_{14} \mathrm{~N}_{4} \mathrm{O}$ & $194-196$ & 56 & 67.66 & 5.26 & 21.05 & 67.62 & 5.23 & 21.04 \\
$4 \mathrm{l}$ & 3"-pyridinyl & $\mathrm{C}_{15} \mathrm{H}_{14} \mathrm{~N}_{4} \mathrm{O}$ & $212-213$ & 46 & 67.66 & 5.26 & 21.05 & 67.63 & 5.22 & 21.03 \\
$4 \mathrm{~m}$ & 4"-pyridinyl & $\mathrm{C}_{15} \mathrm{H}_{14} \mathrm{~N}_{4} \mathrm{O}$ & $233-235$ & 48 & 67.66 & 5.26 & 21.05 & 67.64 & 5.25 & 21.06 \\
$4 \mathrm{n}$ & 2"thienyl & $\mathrm{C}_{14} \mathrm{H}_{13} \mathrm{~N}_{3} \mathrm{OS}$ & $240-241$ & 55 & 61.99 & 4.79 & 15.49 & 61.98 & 4.78 & 15.51 \\
\hline
\end{tabular}

TABLE 2: SPECTRAL DATA OF THE PREPARED COMPOUNDS 4 (a-n)

\begin{tabular}{|c|c|c|}
\hline S. No. & IR spectral data & ${ }^{1}$ H NMR spectral data Chemical shift $(\delta)$ in ppm \\
\hline $4 a$ & 3380( $\left.\mathrm{NH}_{2}\right), 1591(\mathrm{C}=\mathrm{N}), 1503(\mathrm{C}=\mathrm{C})$ & $\begin{array}{c}\text { 3.75-4.0 }\left(9 \mathrm{H}, \mathrm{s}, 3 \mathrm{xOCH}_{3}\right), 5.15\left(2 \mathrm{H}, \mathrm{s},-\mathrm{NH}_{2}\right), 6.45-6.60\left(1 \mathrm{H}, \mathrm{s}, \mathrm{C}-4{ }^{\prime}-\mathrm{H}\right), 7.45(1 \mathrm{H}, \mathrm{s}, \mathrm{C}-5-\mathrm{H}), 6.40(2 \mathrm{H}, \mathrm{S}, \\
\mathrm{C}-2 "-\mathrm{H} \text { and C-6"- }-\mathrm{H}), 2.4\left(3 \mathrm{H}, \mathrm{s}, \mathrm{Ar}-\mathrm{CH}_{3}\right), 2.9\left(3 \mathrm{H}, \mathrm{s}, \mathrm{Ar}-\mathrm{CH}_{3}\right) .\end{array}$ \\
\hline $4 b$ & $3346\left(\mathrm{NH}_{2}\right), 1636(\mathrm{C}=\mathrm{N}), 1578(\mathrm{C}=\mathrm{C})$, & $\begin{array}{c}5.45\left(2 \mathrm{H}, \mathrm{s},-\mathrm{NH}_{2}\right), 6.60\left(1 \mathrm{H}, \mathrm{s}, \mathrm{C}-4^{\prime}-\mathrm{H}\right), 7.35(1 \mathrm{H}, \mathrm{s}, \mathrm{C}-5-\mathrm{H}), 8.03(2 \mathrm{H}, \mathrm{d}, \mathrm{J}=8.0 \mathrm{OH}, \mathrm{C}-3 "-\mathrm{H} \text { and C-5"-H), } \\
7.48(2 \mathrm{H}, \mathrm{d}, \mathrm{J}=8.0 \mathrm{~Hz}, \mathrm{C}-2 "-\mathrm{H} \text { and C-6"- } \mathrm{H}), 2.2\left(3 \mathrm{H}, \mathrm{s}, \mathrm{Ar}-\mathrm{CH}_{3}\right), 2.6\left(3 \mathrm{H}, \mathrm{s}, \mathrm{Ar}-\mathrm{CH}_{3}\right) .\end{array}$ \\
\hline $4 c$ & $\begin{array}{l}3332\left(\mathrm{NH}_{2}\right), 1610(\mathrm{C}=\mathrm{N}), 1570( \\
\left.\mathrm{C}=\mathrm{C}), 1178-\mathrm{N}\left(\mathrm{CH}_{3}\right)_{2}\right)\end{array}$ & $\begin{array}{c}3.10\left(6 \mathrm{H}, \mathrm{s},-\mathrm{N}\left(\mathrm{CH}_{3}\right)_{2}\right), 5.20\left(2 \mathrm{H}, \mathrm{s},-\mathrm{NH}_{2}\right), 7.2(1 \mathrm{H}, \mathrm{s}, \mathrm{C}-5-\mathrm{H}), 6.61\left(1 \mathrm{H}, \mathrm{s}, \mathrm{C}-4{ }^{\prime}-\mathrm{H}\right), 8.12(2 \mathrm{H}, \mathrm{d}, \mathrm{J}=8.5 \mathrm{~Hz} \text {, } \\
\mathrm{C}-3 "-\mathrm{H}^{-} \text {and C-5"-H), } 6.78\left(2 \mathrm{H}, \mathrm{d}, \mathrm{J}=8.5 \mathrm{~Hz}, \mathrm{C}-2 "-\mathrm{H} \text { and C-6"-H), } 2.65\left(3 \mathrm{H}, \mathrm{s}, \mathrm{Ar}-\mathrm{CH}_{3}\right), 2.9\left(3 \mathrm{H}, \mathrm{s}, \mathrm{Ar}-\mathrm{CH}_{3}\right) .\right.\end{array}$ \\
\hline $4 d$ & $3335\left(\mathrm{NH}_{2}\right), 1597(\mathrm{C}=\mathrm{N}), 1520(\mathrm{C}=\mathrm{C})$ & $\begin{array}{c}2.46\left(3 \mathrm{H}, \mathrm{s}, \mathrm{Ar}-\mathrm{CH}_{3}\right), 5.25\left(2 \mathrm{H}, \mathrm{s},-\mathrm{NH}_{2}\right), 6.67\left(1 \mathrm{H}, \mathrm{s}, \mathrm{C}-\mathrm{-}^{\prime}-\mathrm{H}\right), 7.45(1 \mathrm{H}, \mathrm{s}, \mathrm{C}-5-\mathrm{H}), 8.06(2 \mathrm{H}, \mathrm{d}, \mathrm{J}=8.0 \mathrm{~Hz}, \mathrm{C}- \\
3^{\prime \prime}-\mathrm{H} \text { and C-5"-H), } 7.36\left(2 \mathrm{H}, \mathrm{d}, \mathrm{J}=8.0 \mathrm{~Hz}, \mathrm{C}-2^{\prime}-\mathrm{H} \text { and C-6"-H), 2.15(3H,s, Ar-CH } \mathrm{CH}_{3}\right), 2.25\left(3 \mathrm{H}, \mathrm{s}, \mathrm{Ar}-\mathrm{CH}_{3}\right) .\end{array}$ \\
\hline $4 e$ & $\begin{array}{l}3326\left(\mathrm{NH}_{2}\right), 1605(\mathrm{C}=\mathrm{N}), 1525(\mathrm{C}=\mathrm{C}) \\
1372(\mathrm{C}-\mathrm{N}), 892(\mathrm{C}-\mathrm{Cl})\end{array}$ & $\begin{array}{c}5.78\left(2 \mathrm{H}, \mathrm{s},-\mathrm{NH}_{2}\right), 6.62\left(1 \mathrm{H}, \mathrm{s}, \mathrm{C}-4^{\prime}-\mathrm{H}\right), 7.62(1 \mathrm{H}, \mathrm{s},-\mathrm{C}-3 "-\mathrm{H}), 7.54(1 \mathrm{H}, \mathrm{d}, \mathrm{J}=8.5 \mathrm{~Hz}, \mathrm{C}-5 "-\mathrm{H}), 7.41(1 \mathrm{H}, \mathrm{d}, \\
\mathrm{J}=8.5 \mathrm{~Hz}, \mathrm{C}-6 "-\mathrm{H}), 7.35(1 \mathrm{H}, \mathrm{s}, \mathrm{C}-5-\mathrm{H}), 2.4\left(3 \mathrm{H}, \mathrm{s}, \mathrm{Ar}-\mathrm{CH}_{3}\right), 2.9\left(3 \mathrm{H}, \mathrm{s}, \mathrm{Ar}-\mathrm{CH}_{3}\right) .\end{array}$ \\
\hline $4 f$ & $3328\left(\mathrm{NH}_{2}\right), 1632(\mathrm{C}=\mathrm{N}), 1515(\mathrm{C}=\mathrm{C})$ & 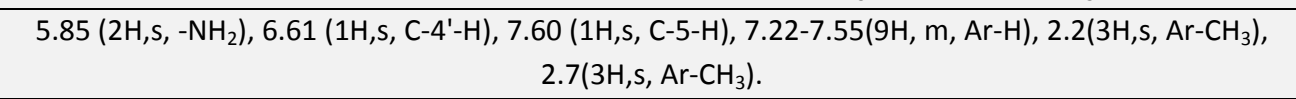 \\
\hline $4 \mathrm{~g}$ & $\begin{array}{l}3414\left(\mathrm{NH}_{2}\right), 1598(\mathrm{C}=\mathrm{N}), 1503(\mathrm{C}=\mathrm{C}) \\
1366(\mathrm{C}-\mathrm{N}), 1225(\mathrm{C}-\mathrm{O}-\mathrm{C})\end{array}$ & $\begin{array}{c}\left.\text { 3.87 (3H, s, C-4"- }-\mathrm{OCH}_{3}\right), 5.11\left(2 \mathrm{H}, \mathrm{s},-\mathrm{NH}_{2}\right), 7.07(2 \mathrm{H}, \mathrm{d}, \mathrm{J}=8.5 \mathrm{~Hz}, \mathrm{C}-3 \text { "and 5"-H), } 7.37(1 \mathrm{H}, \mathrm{s}, \mathrm{C}-5-\mathrm{H}) \text {, } \\
6.51\left(1 \mathrm{H}, \mathrm{s}, \mathrm{C}-4^{\prime}-\mathrm{H}\right), 8.05\left(2 \mathrm{H}, \mathrm{d}, \mathrm{J}=8.5 \mathrm{~Hz}, \mathrm{C}-2^{\prime \prime} \text { and 6"-H), 2.35(3H, s, Ar-CH } \text { 6 }_{3}\right), 2.7\left(3 \mathrm{H}, \mathrm{s}, \mathrm{Ar}-\mathrm{CH}_{3}\right) .\end{array}$ \\
\hline $4 \mathrm{~h}$ & $\begin{array}{c}3320\left(\mathrm{NH}_{2}\right), 1597(\mathrm{C}=\mathrm{N}), 1556(\mathrm{C}=\mathrm{C}) \\
1354(\mathrm{C}-\mathrm{N}), 1261(\mathrm{C}-\mathrm{O}-\mathrm{C})\end{array}$ & $\begin{array}{c}5.21\left(2 \mathrm{H}, \mathrm{s},-\mathrm{NH}_{2}\right), 3.75-4.0\left(6 \mathrm{H}, \mathrm{s}, 2 \times \mathrm{OCH}_{3}\right), 7.19\left(1 \mathrm{H}, \mathrm{S}, \mathrm{C}-2^{\prime \prime}-\mathrm{H}\right), 7.94(2 \mathrm{H}, \mathrm{dd}, \mathrm{J}=8.5 \mathrm{~Hz}, \mathrm{~J}=8.5 \mathrm{~Hz}, \mathrm{C}-3 " \\
\text { and 5"-H), } 6.63\left(1 \mathrm{H}, \mathrm{s}, \mathrm{C}-4^{\prime}-\mathrm{H}\right), 7.0(1 \mathrm{H}, \mathrm{s}, \mathrm{C}-5-\mathrm{H}), 2.35\left(3 \mathrm{H}, \mathrm{s}, \mathrm{Ar}-\mathrm{CH}_{3}\right), 2.7\left(3 \mathrm{H}, \mathrm{s}, \mathrm{Ar}-\mathrm{CH}_{3}\right) .\end{array}$ \\
\hline $4 \mathrm{i}$ & $\begin{array}{l}3318\left(\mathrm{NH}_{2}\right), 1599(\mathrm{C}=\mathrm{N}), 1510(\mathrm{C}=\mathrm{C}) \\
1350(\mathrm{C}-\mathrm{N}), 1219(\mathrm{C}-\mathrm{F})\end{array}$ & $\begin{array}{c}5.21\left(2 \mathrm{H}, \mathrm{s},-\mathrm{NH}_{2}\right), 7.19\left(2 \mathrm{H}, \mathrm{dd}, \mathrm{J}=8.5 \mathrm{~Hz}, \mathrm{C}-2 " \text { and 6"-H), } 6.60\left(1 \mathrm{H}, \mathrm{s}, \mathrm{C}-4^{\prime}-\mathrm{H}\right), 8.2(2 \mathrm{H}, \mathrm{dd}, \mathrm{J}=8.5 \mathrm{~Hz}, \mathrm{C}-\right. \\
3^{\prime \prime} \text { and 5"-H), } 7.25(1 \mathrm{H}, \mathrm{s}, \mathrm{C}-5-\mathrm{H}), 2.4\left(3 \mathrm{H}, \mathrm{s}, \mathrm{Ar}-\mathrm{CH}_{3}\right), 2.8\left(3 \mathrm{H}, \mathrm{s}, \mathrm{Ar}-\mathrm{CH}_{3}\right) .\end{array}$ \\
\hline $4 \mathrm{j}$ & $\begin{array}{l}3370\left(\mathrm{NH}_{2}\right), 1645(\mathrm{C}=\mathrm{N}), 1557(\mathrm{~N}=\mathrm{O}, \\
\text { asymmetric) }\end{array}$ & $\begin{array}{c}5.22\left(2 \mathrm{H}, \mathrm{s},-\mathrm{NH}_{2}\right), 6.64-6.65\left(1 \mathrm{H}, \mathrm{s}, \mathrm{C}-4{ }^{\prime}-\mathrm{H}\right), 7.35(1 \mathrm{H}, \mathrm{s}, \mathrm{C}-5-\mathrm{H}), 7.79\left(2 \mathrm{H}, \mathrm{d}, \mathrm{J}=8.0 \mathrm{~Hz}, \mathrm{C}-2^{\prime \prime} \text { and 6"-H }\right) \text {, } \\
8.34(2 \mathrm{H}, \mathrm{d}, \mathrm{J}=8.0 \mathrm{~Hz}, \mathrm{C}-3 \text { " and 5"- }-\mathrm{H}), 2.2\left(3 \mathrm{H}, \mathrm{s}, \mathrm{Ar}-\mathrm{CH}_{3}\right), 2.6\left(3 \mathrm{H}, \mathrm{s}, \mathrm{Ar}-\mathrm{CH}_{3}\right) .\end{array}$ \\
\hline $4 \mathrm{k}$ & $\begin{array}{c}3425,3238\left(\mathrm{NH}_{2}\right), 1656(\mathrm{C}=\mathrm{N}), 1510 \\
(\mathrm{C}=\mathrm{C})\end{array}$ & 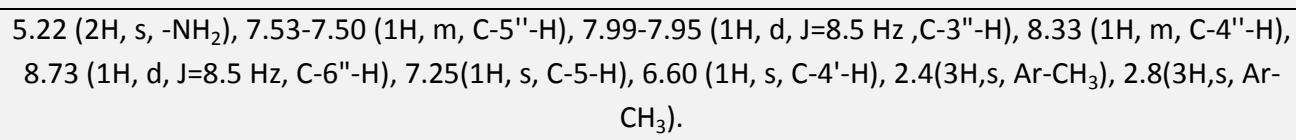 \\
\hline 41 & $\begin{array}{c}3415\left(\mathrm{NH}_{2}\right), 1645(\mathrm{C}=\mathrm{N}), 1512(\mathrm{C}=\mathrm{C}) \\
1359(\mathrm{C}-\mathrm{N})\end{array}$ & $\begin{array}{c}5.3\left(2 \mathrm{H}, \mathrm{s},-\mathrm{NH}_{2}\right), 7.53-7.50(1 \mathrm{H}, \mathrm{m}, \mathrm{C}-5 "-\mathrm{H}), 6.62\left(1 \mathrm{H}, \mathrm{s}, \mathrm{C}-4^{\prime}-\mathrm{H}\right), 7.25(1 \mathrm{H}, \mathrm{s}, \mathrm{C}-5-\mathrm{H}), 8.33(1 \mathrm{H}, \mathrm{d}, \mathrm{J}=8.0 \\
\left.\mathrm{Hz}, \mathrm{C}-4^{\prime \prime}-\mathrm{H}\right), 7.4\left(1 \mathrm{H}, \mathrm{s}, \mathrm{C}-2^{\prime}-\mathrm{H}\right), 8.73(3 \mathrm{H}, \mathrm{d}, \mathrm{J}=8.0 \mathrm{~Hz}, \mathrm{C}-6 "-\mathrm{H}), 2.4\left(1 \mathrm{H}, \mathrm{s}, \mathrm{Ar}-\mathrm{CH}_{3}\right), 2.8\left(3 \mathrm{H}, \mathrm{s}, \mathrm{Ar}-\mathrm{CH}_{3}\right) .\end{array}$ \\
\hline $4 m$ & $3418\left(\mathrm{NH}_{2}\right), 1575(\mathrm{C}=\mathrm{N}), 1526(\mathrm{C}=\mathrm{C})$ & $\begin{array}{c}5.32\left(2 \mathrm{H}, \mathrm{s},-\mathrm{NH}_{2}\right), 6.55-6.54\left(1 \mathrm{H}, \mathrm{s}, \mathrm{C}-4{ }^{\prime} \mathrm{H}\right), 7.25(1 \mathrm{H}, \mathrm{s}, \mathrm{C}-5-\mathrm{H}), 7.46(2 \mathrm{H}, \mathrm{d}, \mathrm{J}=8.5 \mathrm{~Hz}, \mathrm{C}-3 " \mathrm{H} \text { and 5"H), } \\
7.58(2 \mathrm{H}, \mathrm{d}, \mathrm{J}=8.2 \mathrm{~Hz} \mathrm{C}-2 " \mathrm{H} \text { and } 6 " \mathrm{H}), 2.4\left(3 \mathrm{H}, \mathrm{s}, \mathrm{Ar}-\mathrm{CH}_{3}\right), 2.7\left(3 \mathrm{H}, \mathrm{s} \mathrm{Ar}-\mathrm{CH}_{3}\right) .\end{array}$ \\
\hline $4 n$ & $\begin{array}{l}3405\left(\mathrm{NH}_{2}\right), 1565(\mathrm{C}=\mathrm{N}), 1516(\mathrm{C}-\mathrm{C}) \\
1360(\mathrm{C}-\mathrm{N}), 670(\mathrm{C}-\mathrm{S})\end{array}$ & $\begin{array}{c}5.3\left(2 \mathrm{H}, \mathrm{s},-\mathrm{NH}_{2}\right), 6.55-6.58\left(1 \mathrm{H}, \mathrm{s}, \mathrm{C}-4{ }^{\prime} \mathrm{H}\right), 7.32(1 \mathrm{H}, \mathrm{s}, \mathrm{C}-5-\mathrm{H}), 7.16-7.12(1 \mathrm{H}, \mathrm{t}, \mathrm{C}-4 " \mathrm{H}), 7.26(1 \mathrm{H}, \mathrm{d} \\
\mathrm{J}=6 \mathrm{~Hz}, \mathrm{C}-3 " \mathrm{H}), 7.46(1 \mathrm{H}, \mathrm{d}, \mathrm{J}=8 \mathrm{~Hz}, \mathrm{C}-5 " \mathrm{H}), 2.3\left(3 \mathrm{H}, \mathrm{s}, \mathrm{Ar}-\mathrm{CH}_{3}\right), 2.5\left(3 \mathrm{H}, \mathrm{s}, \mathrm{Ar}_{-}-\mathrm{CH}_{3}\right) .\end{array}$ \\
\hline
\end{tabular}

Anticancer activity: The synthesized pyrimidines have been screened for anticancer activity on prostate cancer cell lines ( DU-145) using MTT based cytotoxicity assay ${ }^{17}$. The required cell proliferation assay kit was obtained from Roche Applied Sciences, Germany. The results (mean O.D. \pm SD) obtained from quadruplicate wells were used in calculation to determine the $\mathrm{IC}_{50}$ of the test compounds.
The percent inhibition is then calculated from the formula:

$\%$ inhibition $=$ Control O.D. - Sample O.D. $\times 100$

Control O.D.

The results are presented in Table $\mathbf{3}$ for anticancer activity. 
TABLE 3: ANTICANCER ACTIVITY OF PYRIMIDINES DERIVATIVES ON DU-145 CELL LINES

\begin{tabular}{cc}
\hline Compound Code & Percent inhibition at $50 \mu \mathrm{g} / \mathrm{mL}$ \\
\hline $4 \mathrm{a}$ & 15.24 \\
$4 \mathrm{~b}$ & 43.62 \\
$4 \mathrm{c}$ & 12.12 \\
$4 \mathrm{~d}$ & 13.5 \\
$4 \mathrm{e}$ & 39.52 \\
$4 \mathrm{f}$ & 21.36 \\
$4 \mathrm{~g}$ & 9.34 \\
$4 \mathrm{~h}$ & 16.22 \\
$4 \mathrm{i}$ & 15.24 \\
$4 \mathrm{j}$ & 8.26 \\
$4 \mathrm{k}$ & 12.22 \\
$4 \mathrm{l}$ & 11.46 \\
$4 \mathrm{~m}$ & 12.62 \\
$4 \mathrm{n}$ & 23.48 \\
\hline
\end{tabular}

RESULTS AND DISCUSSION: The title compounds 2amino-4-(2', 5'-dimethyl-3'-furyl)-6-(aryl) pyrimidines $(4 a-n)$ were synthesized in good yields (scheme-I). Some of the compounds have significant anticancer activity against the cell lines (DU-145). Out of all the compounds, $\mathbf{4 b}$ containing 4-chlorophenyl substitution on $6^{\text {th }}$ position of pyrimidine nucleus showed maximum activity, closely followed by $4 \mathrm{e}$ containing thiophene ring. These compounds also need to be tested on other cancer cell lines in order to predict their activity and therapeutic usefulness.

ACKNOWLEDGEMENT: The authors are thankful to Dr. Trimurtulu, Vice-President, LAILA IMPEX Industries Ltd., Vijayawada, India for providing IR, ${ }^{1} \mathrm{H} N M R$ spectral data.

\section{REFERENCES:}

1. Ozair A, Mohd I and Khan SA: Synthesis and biological activity of some pyrimidine derivatives. Indian J. Heterocyclic Chem. 2005; 14: 293.

2. Amir $M$, Javed $S A$ and Harishkumar: Pyrimidine as antiinflammatory agent: A review. Indian j. pharm. sci. 2007; 69(3): 337.
3. Anjani S, Smruti L, Sejal S and Ghanshyam P: Chalcones, pyrazolines and aminopyrimidines as antibacterial agents. Ind. J. chem. 2009; 48b: 1442-1446.

4. Anjani S, Kishor K, Ana C, Marina S, Irini D and Athina G: Synthesis of some new S-triazine based chalcones and their derivatives as potent antimicrobial agents. Eur. J. Med. Chem. 2010; 45: 510-518.

5. Dana $\mathrm{H}$, Antonin $\mathrm{H}$, Milena $\mathrm{M}$, Graciela $A$, Robert $\mathrm{S}$, Erik $\mathrm{D}$ and Jan B: Synthesis and antiviral activity of 2,4-diamino-5-cyano-6[2-(phosphonomethoxy) ethoxy] pyrimidine and related compounds. Bioorg. Med. Chem. 2004; 12(12): 3197-3202.

6. Sham MS, Monica D, Reshma R, Rakesh S and Ram R: Synthesis, anti-inflammatory and analgesic avtivity evaluation of some new pyrimidine derivatives. Ind. J. Chem. 2009; 49b: 273-281.

7. Rahaman Sk, Rajendraprasad Y, Phanikumar and Bharatkumar: Synthesis and antihistaminic activity of some novel pyrimidines. Saudi Pharmaceutical Journal, 2009; 17 (3): 259-263.

8. Braulio I, Fabián $\mathrm{O}$, Jairo $\mathrm{Q}$, Rodrigo $\mathrm{A}$, Manuel $\mathrm{N}$ and Justo $\mathrm{C}$ : Microwave induced synthesis of novel 8,9-dihydro-7Hpyrimido[4,5-b][1,4]diazepines as potential antitumour agents. Eur. J. Med. Chem. 2008; 43(9): 1955-1962.

9. Silvana R, Drazenka S, Tatjana G, Andreja M, Antonija H, Jan B, Erik $D$ and Mladen $M$ : Synthesis and Antitumor Activities of Novel Pyrimidine Derivatives of 2,3-0,0-Dibenzyl-6-deoxy-Iascorbic Acid and 4,5-Didehydro-5,6- dideoxy-I-ascorbic Acid. J. Med. Chem. 2000, 43 (25), 4806-4811.

10. Lukevits E. and Demicheva L: Biological activity of furan derivatives. Chem. het. Comp. 1993; 29(3): 243-267.

11. Logoglu E, Yilmaz $M$, Katircioglu $H$, Yakut $M$ and Mercan S: Synthesis and biological activity studies of furan derivatives. Med.Chem.Res.2010; 19(5): 490-497.

12. Gupta DP, Ahmad S and Shanker: Synthesis, anthelmintic and antiacetyl cholinesterase activity of newer furan derivatives. Indian J. Pharma. Sci. 1988; 50(2): 98-100.

13. Rajendraprasad $Y$, Ravikumar $P$ and Ramesh B: Synthesis and antidepressant activity of somenew 3-(2"-hydroxy naphthalen1"-yl)-5-phenyl-2-isoxazolines. Int. J. Chem. Sci.: 2007; 5(2): 542-548.

14. Rajendra Prasad Y, Lakshmana Rao A, Prasoona L, Murali K and Ravi Kumar P: Synthesis and antidepressant activity of some 1, 3, 5-triphenyl-2-pyrazolines and 3-(2"-hydroxy naphthalen-1"yl)-1,5-diphenyl-2-pyrazolines. Bioorg. Med. Chem. Lett. 2005; 15(22): 5030-5034.

15. Amit RT, Dipti KD, Naresh RR and Viresh HS: Synthesis and biological evaluation of some new pyrimidines via a novel chalcone series, Arkivoc. 2008; (xi): 131-141.

16. Rajendraprasad $Y$, Praveenkumar $P$ and Ravikumar $P$, Synthesis and biological evaluation of some new 2,4,6-trisubstitued pyrimidines, Oriental J. Chem. 2007; 23(3): 1069-1072.

17. Mosmann T, Rapid colorimetric assay for cellular growth and survival: application to proliferation and cytotoxicity assays. J.Immunol Methods. 1983; 65: 55. 